\title{
RASTREAMENTO DE INTERAÇÕES EM LABORATÓRIOS EDUCACIONAIS NOS MUNDOS VIRTUAIS 3D PA̧RA IDENTIFICAÇÃO DE ENGAJAMENTO.
}

\author{
Leandro Rosniak Tibola - PPGIE/UFRGS - URI-FW (tibola@uri.edu.br) \\ Liane M. Rockenbach Tarouco - PPGIE/UFRGS (liane@ penta.ufrgs.br)
}

\begin{abstract}
Resumo: O equilíbrio entre teoria e prática sempre foi um desafio histórico na educação, bem como a identificação do engajamento dos alunos nas atividades acadêmicas. Deseja-se o equilíbrio teórico-prático para ampliar os conhecimentos, habilidades e atitudes; e a melhora do engajamento para impulsionar o desempenho dos estudantes. Os laboratórios virtuais desenvolvidos em mundos virtuais 3D são uma alternativa para este desafio, permitindo medir o nível de engajamento do estudante nas experiências práticas. Este artigo apresenta o desenvolvimento de laboratórios em mundos virtuais 3D para as aprendizagens experiencial e significativa, armazenando as interações do aluno com o experimento, permitindo a identificação de seu nível de engajamento.
\end{abstract}

Palavras-chaves: Aprendizagem experiencial. Aprendizagem significativa. Laboratórios virtuais. Mundos virtuais 3D. Engajamento.

\section{TRACKING OF INTERACTIONS IN EDUCATIONAL LABS WITHIN 3D VIRTUAL WORLDS TO IDENTIFICATION OF ENGAGEMENT.}

Abstract: The balance between theoretical and practical lessons has always been a challenge to education, like identify the engagement level of students in academicals activities. We seek for theoretical and practical balance to expand the knowledge, abilities and attitudes; and to improve the engagement to promote the performance of students. The virtual labs developed within $3 d$ virtual worlds are a choice to solve this, allowing to measure student's engagement level in practical experiences. This paper presents the virtual labs development within $3 D$ virtual worlds for both experimental and meaningful learning, recording the student's interactions with labs, allow identify student engagement level.

Keywords: Experiential learning. Meaningful learning. Virtual labs. 3D virtual worlds. Engagement.

\section{Introdução}

Quando o ser humano realiza qualquer atividade, o faz usando as ciências da natureza e as leis da Física, da Química e da Biologia, todas embasadas pela Matemática. Em muitos casos, a utilização destas leis é intuitiva ou inconsciente. Porém, quando a pessoa está se prepara como profissional, deve dominar estas leis.

No caso da Física, o método de ensino tradicional baseia a transposição destes conhecimentos através livros didáticos e exames de vestibulares como se estes fossem as únicas alternativas para orientar as aulas. O resultando desta didática é, frequentemente, um ensino de respostas sem a formulação de perguntas, a qual ignora uma característica fundamental da ciência: a sua dimensão investigativa (MEC, 2006).

Neste sentido, MEC (2015) sugere que o ensino de Física deixe de se concentrar na simples memorização de fórmulas ou na repetição automatizada de procedimentos, para ganhar a consciência de que é preciso dar um significado à Física, explicitando seu 
sentido já no momento do aprendizado, alterando assim a referência tradicional de "o que ensinar de Física" passando "para que ensinar Física".

A partir disto, surgem alguns questionamentos: Como tornar os conceitos abstratos de Física mais concretos através de situações práticas? Como verificar as ações efetuadas pelo aprendiz neste formato? É possível identificar indícios da aprendizagem nesta proposta? Para encontrar respostas para estas questões, necessitamos de abordagens que sejam voltadas para o "fazer", para o "concreto", para o "significativo".

Como possibilidade, temos a proposta de Kolb (1984), onde o conhecimento é fruto da combinação da compreensão e da transformação da experiência. Em adição, o uso da tecnologia para engajar os estudantes numa aprendizagem ativa, construtiva, intencional, autêntica e cooperativa sugerida por Jonassen et al. (2008), as quais podem embasar a passagem do conceitual para o prático. Ambas são descritas na seção 2.

Para aplicar estas abordagens, a construção de Laboratórios Virtuais (LV) permite associar os conceitos teóricos da Física a aplicações práticas e significativas através de objetos virtuais que possuem funcionamento dinâmico. Quando desenvolvidos dentro de Mundos Virtuais 3D (MV3D), os LV apresentam as vantagens de forte interação entre usuários e objetos, fornecem a sensação de imersão e permitem registrar as ações realizadas no LV e dentro do mundo. Estes conceitos são descritos na seção 2. Já a seção 3 apresenta alguns trabalhos que motivaram a escrita deste artigo. Assim, baseados nestes conceitos, são apresentadas as seguintes propostas:

- A construção de artefatos para laboratórios virtuais dentro de mundos virtuais 3D que promovam a aprendizagem experiencial e que contemplem uma taxonomia adequada são apresentados na seção 4.

- A verificação das ações efetuadas pelo aprendiz no LV, através de sensores que monitorem as ações sobre os objetos e o seu registro em banco de dados é descrita na seção 5 .

A seção 6 encerra artigo com as conclusões. Este trabalho é parte de uma tese em andamento, assim são apresentados os resultados parciais obtidos até o momento.

\section{Fundamentação Teórica}

\subsection{Aprendizagem experiencial}

Segundo Kolb et al. (1999), a Teoria da Aprendizagem Experiencial fornece um modelo holístico do processo de aprendizagem e um modelo multilinear de desenvolvimento, os quais são consistentes com o que se conhece sobre como as pessoas aprendem, crescem e se desenvolvem. Para Kolb (1984), na teoria de aprendizagem experiencial a aprendizagem é "o processo pelo qual o conhecimento é criado através da transformação da experiência, o conhecimento resulta da combinação de compreender e transformar a experiência". O processo de aprendizagem experiencial é composto por quatro estilos de aprendizagem, formando um modelo cíclico aplicável em vários contextos. Os estilos de aprendizagem se desenvolvem como consequência dos fatores hereditários, das experiências anteriores e das condições do meio atual em que o sujeito vive.

Os estilos de aprendizagem estão estruturados sobre um círculo de aprendizagem de quatro estágios. Estas duas camadas, de estilos e de estágios, compõem o Ciclo de Kolb. A camada interna é formada pelos quatro estágios: (a) Experiência Concreta (EC); (b) Observação Reflexiva (OR); (c) Conceituação Abstrata (CA); (d) Experimentação Ativa (EA). A camada externa é formada pelos quatro estilos: (i) Divergência; (ii) Assimilação; (iii) Convergência e (iv) Acomodação (KOLB, 1984). 


\subsection{Aprendizagem Significativa}

O objetivo principal da educação deveria ser engajar os estudantes na aprendizagem significativa e a obrigação primária das escolas deveria ser ajudar os estudantes a aprender como reconhecer e resolver problemas, compreender novos fenômenos, construir modelos mentais deles e, dada uma nova situação, estabelecer objetivos e regular sua própria aprendizagem (aprender a aprender) (JONASSEN et al., 1999).

Para Jonassen et al. (1999), a aprendizagem significativa pode ser vista a partir de 5 atributos : (1) Aprendizagem Ativa (Manipulativa/Observadora): aprendizado resulta de experiências genuínas, pois as pessoas interagem com seu ambiente e manipulam os objetos deste ambiente, observando os efeitos dessas intervenções e construindo suas próprias interpretações do fenômeno e dos resultados da manipulação; (2) Aprendizagem Construtiva (Articulativa/Reflexiva): o aprendiz constroi seu próprio significado para a experiência, uma vez que devem refletir a respeito da sua atividade e de suas observações para aprender a lição que esta atividade tem para ensinar; (3) Aprendizagem Intencional (Reflexiva/Regulatória): quando o aprendiz intenciona atingir um objetivo, ele exige mais de sua capacidade cognitiva, ativa e intencionalmente, buscando este fim; (4) Aprendizagem Autêntica (Complexa/Contextualizada):em vez de experiências abstratas, é necessário ensinar conhecimento e habilidades da vida real, utilizando contextos úteis, novos e diferentes para que os alunos pratiquem usando aqueles conceitos teóricos previamente conhecidos e (5) Aprendizagem Cooperativa (Colaborativa/Conversacional):os alunos trabalham na construção da aprendizagem e do conhecimento construindo comunidades, explorando as habilidades de cada um, enquanto fornecem apoio moral, modelam e observam as contribuições de cada membro.

Para Howland, Jonassen e Marra (2008), os estudantes também podem aprender significativamente interagindo com os mundos virtuais, os quais combinam muitos dos aspectos presentes nos simuladores e nos jogos, uma vez que o mundo virtual parece e funciona semelhantemente com o mundo real.

\subsection{Laboratórios Virtuais}

Conforme Feisel e Rosa (2005), os laboratórios podem ser de três tipos: (1) de desenvolvimento, usados para criação de produtos; (2) de pesquisa, geram a base de conhecimento para outras pesquisas que vão aplica-lo e (3) de educação, objetivam consolidar nos estudantes o conhecimento teórico através prática. Por sua vez, Auer et al. (2003) classificam os laboratórios de acordo com a localização dos estudantes e do tipo de experimento.

No caso específico dos laboratórios educacionais, é necessário avaliar dois aspectos importantes: o custo e a segurança. Devido ao seu custo crescente de instalação, os laboratórios físicos têm sofrido restrições de investimentos desde o fim da Guerra do Vietnã e do projeto de viajem à Lua, segundo Feisel e Rosa (2005). Além da instalação, os laboratórios físicos apresentam custos adicionais: (a) suprimentos e reagentes; (b) manutenção predial; (c) manutenção dos equipamentos; (d) pessoal de apoio, etc.

O incremento da segurança dos laboratórios virtuais em relação aos físicos pode ser medida pela não exposição dos alunos aos agentes nocivos, tais como produtos químicos, corrosivos e biológicos; pelo manuseio de aparelhos perigosos com segurança; sem a necessidade de treinamento. 


\subsection{Mundos Virtuais 3D}

Para Nelson e Erlandson (2012) um mundo virtual é um mundo 3D baseado em computador o qual você pode explorar sozinho ou com outras pessoas. Em um mundo virtual você tanto explora o mundo como você mesmo (com a visão em primeira pessoa) ou é representado por um personagem gerado pelo computador chamado avatar (com a visão em terceira pessoa).

Por sua vez, Klastrup (2003) define mundo virtual como uma representação online persistente que contém a possibilidade de interação síncrona entre usuários e entre o usuário e mundo, dentro das regras de espaço desenvolvidas, como um universo navegável. Nos mundos virtuais você pode se mover, através de representações persistentes do usuário, contrastando com mundos tradicionais da ficção, que são mundos apresentados como habitados por pessoas reais, mas que não são exatamente habitáveis. Assim, a interação nos mundos virtuais é realizada através dos avatares.

De acordo com Santaella (2003), avatar é um cibercorpo inteiramente digital, uma figura gráfica de complexidade variada que empresta sua vida simulada para o transporte de cibernautas para dentro dos mundos paralelos do ciberespaço. Já Mattar (2008) é incisivo ao descrever a presença de uma pessoa no mundo virtual através de um avatar: "você está situado, vê e ouve coisas daquele ponto de vista e então parece estar presente naquele ambiente".

Além dos avatares, os mundos virtuais são compostos por primitivos e objetos. Os primitivos, ou prims, são a menor forma presente no mundo virtual. Os prims são as formas geométricas básicas que permitem a construção de objetos mais complexos, os principais são: cubo, esfera, pirâmide, cilindro, cone, anel. Nos mundos virtuais, objetos tais como carros, casas, joias e até coisas evidentes como cabelo e roupa são construídos com um ou mais prims (SECOND LIFE, 2013).

Em adição as suas características geométricas e visuais, é possível dotar os prims e os objetos com comportamento. A forma mais simples de inserir comportamento nos prims ou objetos é através da Linden Scripting Language ou LSL (SECOND LIFE, 2013). Os scripts LSL permitem comportamentos ativos, como quando o objeto é "tocado" pelo avatar, o script executa determinada ação ou reativos, como quando o avatar se aproxima do objeto ou quando o script é criado no objeto. Este trabalho utilizou o mundo virtual Open Simulator ou OpenSim (OPENSIM, 2013).

\subsection{Mineração de Dados Educacionais}

A mineração de dados é uma das fases de um processo mais amplo, chamado de Descoberta de Conhecimento em Base de Dados (DCBD), este artigo foca especificamente na MDE. Segundo IEDMS (2015) a Mineração de Dados Educacionais - MDE (Educational Data Mining - EDM) é uma disciplina emergente, preocupada com o desenvolvimento de métodos para explorar, o único e cada vez maior, conjunto de dados em larga escala que vêm de contextos educacionais e utilizar esses métodos para entender melhor os alunos e as condições em que eles aprendem.

Segundo Costa et al. (2012), a área emergente de Mineração de Dados Educacionais procura desenvolver ou adaptar métodos e algoritmos de mineração existentes, de tal modo que se prestem a compreender melhor os dados em contextos educacionais, produzidos principalmente por estudantes e professores, considerando os ambientes nos quais eles interagem, tais como AVAs, Sistemas Tutores Inteligentes (STIs), entre outros. Com tais métodos visa-se, por exemplo, entender melhor o estudante no seu processo de aprendizagem, analisando-se sua interação com o ambiente. 


\subsection{Learning Analytics}

Segundo Ferguson (2012) Learning Analytics é a medição, coleta, análise e o relatório de dados sobre alunos e seus contextos, para fins de compreensão e otimização da aprendizagem e dos ambientes em que ocorre.

Já para Siemens (2010) Learning Analytics é a utilização inteligente de dados, de dados produzidos pelos alunos, juntamente com modelos de análise, a fim de descobrir informações e conexões sociais, objetivando diagnosticar e aconselhar o estudante sobre sua aprendizagem.

Assim, Learning Analytics precisa usar todos os recursos disponíveis no processo educacional, quer sejam equipamentos, pessoas, materiais, processos e dados. Agrupamento e classificação de informações permite mapear os alunos, identificando aqueles que têm alguma deficiência acadêmica. Uma vez identificado o aluno em risco, o sistema de monitoramento pode enviar uma mensagem para ele, apontando uma direção a seguir ou oferecendo apoio. Em casos específicos, o sistema de monitoramento poderia ativar uma ação humana, por meio de conselheiro. $O$ conselheiro pode assim estabelecer um contato pessoal com o aluno, buscando identificar a raiz dos problemas e orientar o aluno para uma solução.

\section{Trabalhos Relacionados}

As pesquisas que contribuíram para a realização deste trabalho envolvem aspectos como: a investigação dos recursos de interação entre o indivíduo e o mundo virtual (TIBOLA, TAROUCO e PASSERINO, 2013); as diversas abordagens para a construção de laboratórios virtuais (SCHAF, 2011; VOSS et al., 2012; AMARAL , ÁVILA e TAROUCO, 2012; ZEDNIK et al., 2012; ÁVILA, AMARAL e TAROUCO, 2013; GREIS, REATEGUI e MARQUES, 2013); e a procura por uma taxonomia para estes ambientes (MYLLER et al., 2009; AMARAL et al., 2011; ÁVILA et al., 2012).

Alguns trabalhos apresentam pontos de interesses em comum com este artigo: o uso de Learning Analytics para potencializar os dados do processo de ensinoaprendizagem e sua utilização para estudar o aprendizado do aluno (WORSLEY e BLIKSTEIN, 2013); a coleta de informações em mundos virtuais para medir o engajamento e identificar as necessidades dos alunos (CAMILLERI et al., 2013); a descoberta de fluxos de aprendizagem e a verificação da sua conformidade por meio de técnicas de mineração de dados e Learning Analytics (FERNÁNDEZ-GALLEGO et al., 2013; CRUZ-BENITO et al., 2014); o estudo de como a Learning Analytics pode ser usada para melhorar a retenção em ambientes virtuais de aprendizagem (WOLFF et al., 2013); o suporte aos professores no processo de avaliação da aprendizagem nos mundos virtuais (KICKMEIER-RUST; ALBERT, 2013); e a verificação da construção de conhecimento dentro dos contextos social e cultural a partir da Social Learning Analytics (FERGUSON; SHUM, 2012).

\section{A construção dos Laboratórios Virtuais}

Este trabalho propõe que nos mundos virtuais, o laboratório como um todo e os objetos que constituem os laboratórios em específico, sejam projetados para promover a aprendizagem ativa e experiencial. Para isto, eles precisam despertar o maior nível de engajamento possível no estudante. Como forma de identificar o nível de engajamento do estudante, foi usada a taxonomia de engajamento estendida, proposta por Myller et al. (2009), a qual classifica as variações no engajamento proveniente da utilização de ferramentas de visualização, analisando a aplicabilidade da taxonomia na descrição das diferenças no processo de colaboração quando a visualização é utilizada. A Figura 1 apresenta esta taxonomia. 


\begin{tabular}{|c|l|l|}
\hline 1 & $\begin{array}{l}\text { Sem } \\
\text { visualização }\end{array}$ & $\begin{array}{l}\text { Nenhuma visualização é apresentada, mas somente material em } \\
\text { formato textual. }\end{array}$ \\
\hline 2 & Visualização & A visualização é apresentada sem nenhuma interação. \\
\hline 3 & $\begin{array}{l}\text { Visualização } \\
\text { controlada }\end{array}$ & $\begin{array}{l}\text { A visualização é apresentada e o estudante controla o fluxo da } \\
\text { visualização. }\end{array}$ \\
\hline 4 & Entrando dados & $\begin{array}{l}\text { O estudante informa dados para um programa ou parâmetros para } \\
\text { um método antes ou durante a execução. }\end{array}$ \\
\hline 5 & Respondendo & $\begin{array}{l}\text { A visualização é acompanhada por questionamentos, os quais estão } \\
\text { relacionados ao contexto da visualização. }\end{array}$ \\
\hline 6 & Alterando & $\begin{array}{l}\text { A alteração da visualização é permitida durante sua apresentação, } \\
\text { através da sua manipulação direta. }\end{array}$ \\
\hline 7 & Modificando & $\begin{array}{l}\text { A modificação da visualização é realizada antes de ser apresentada, } \\
\text { através da alteração no código fonte ou conjunto de parâmetros, } \\
\text { por exemplo. }\end{array}$ \\
\hline 8 & Construindo & $\begin{array}{l}\text { A visualização é criada interativamente pelo estudante através da } \\
\text { construção de componentes tais como texto e figuras geométricas. }\end{array}$ \\
\hline 9 & Apresentação & $\begin{array}{l}\text { A visualização é apresentada e explicada para outros para feedback } \\
\text { e discussão. }\end{array}$ \\
\hline 10 & Revisando & $\begin{array}{l}\text { As visualizaç̃̃es são revisadas a fim de fornecer comentários, } \\
\text { sugestões e feedback sobre a própria visualização. }\end{array}$ \\
\hline
\end{tabular}

Figura 1: Taxonomia de engajamento estendida de Myller et al. (2009).

No contexto da experiência como um todo, foi prevista no projeto do Laboratório Virtual, uma área de conhecimento prévio aos experimentos, na qual o estudante poderá ter contato com os conceitos envolvidos no laboratório ou se ele já os teve, poderá revisá-los. Esta área pode conter textos explicativos, imagens elucidativas, animações e vídeos com exemplos e painéis com links para a Internet, uma vez que esta estratégia está alinhada a ideia de subsunçores de Ausubel (MOREIRA, 1999). Na Figura 2 (A) podemos ver algumas opções da área pré experiência, que permite o conhecimento e/ou revisão do tema.

Da mesma forma, ao concluir a experiência, o estudante é convidado a responder algumas perguntas sobre os conhecimentos desenvolvidos durante a realização das experiências. Um exemplo destas perguntas é apresentado na Figura 2 (B). Como o mundo virtual 3D não é sequencial, não há obrigatoriedade da passagem do aluno por estas áreas, mas é aconselhada aos novatos visitarem a área de conhecimento prévio ou revisão e para todos os estudantes a visita à área pós-experiência.

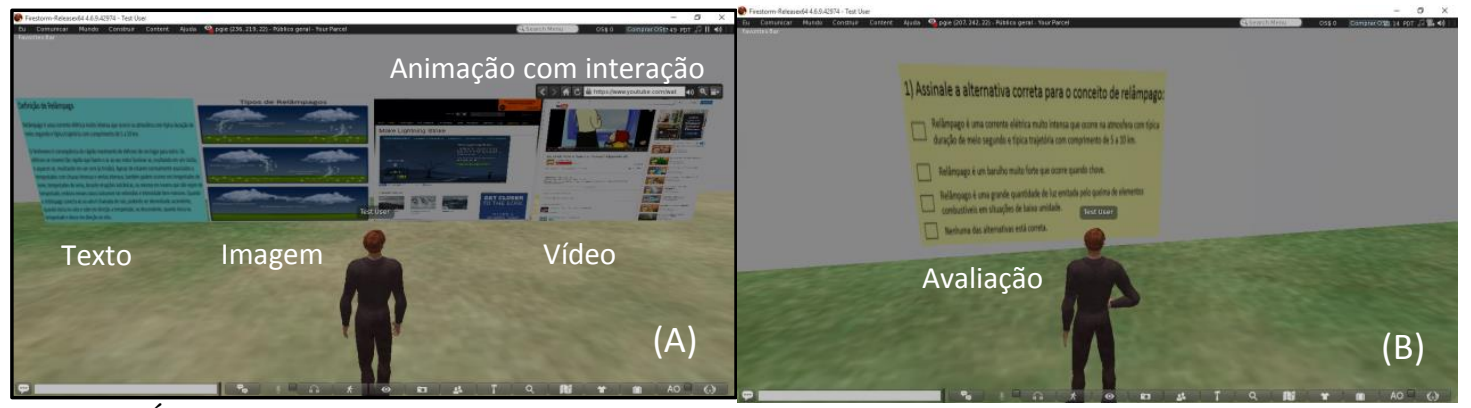

Figura 2: Área anterior (A) e área posterior à experiência (B).

\section{Monitoramento das Ações do Estudante}

A fim de identificar com qual nível de engajamento o estudante pode ser associado, faz-se necessário o rastreamento de suas ações quando das interações com os objetos e na realização dos experimentos. Para atingir este fim, foram desenvolvidos objetos chamados de sensores, os quais possuem código LSL embutido, que permitem identificar a presença do avatar e monitorar quais foram as ações realizadas por ele durante a interação com o objeto ou se não houveram ações devido a falta de interação. Os sensores podem ser de dois tipos: (I) sensores geográficos e (II) sensores de interação.

Os sensores geográficos possuem um funcionamento transparente para o aluno, pois ele não percebe a atuação dos sensores, uma vez que estes executam em segundo 
plano. Em termos de identificação visual, neste trabalho decidiu-se identificar os sensores geográfico com o símbolo de wireless.

Ainda, os sensores geográficos permitem verificar posicionamento do aluno dentro do mundo virtual, se ele entrou e/ou saiu de um prédio ou sala, se passou por determinado lugar, se parou ou não, por quanto tempo, conforme a necessidade de registro.

Por sua vez, os sensores de interação podem ser objetos com este objetivo específico ou podem ser partes do código LSL presentes em outros objetos, os quais possuem a função de identificar quais as interações ocorreram entre o avatar e o objeto. A Figura 3 apresenta o experimento de relâmpagos.

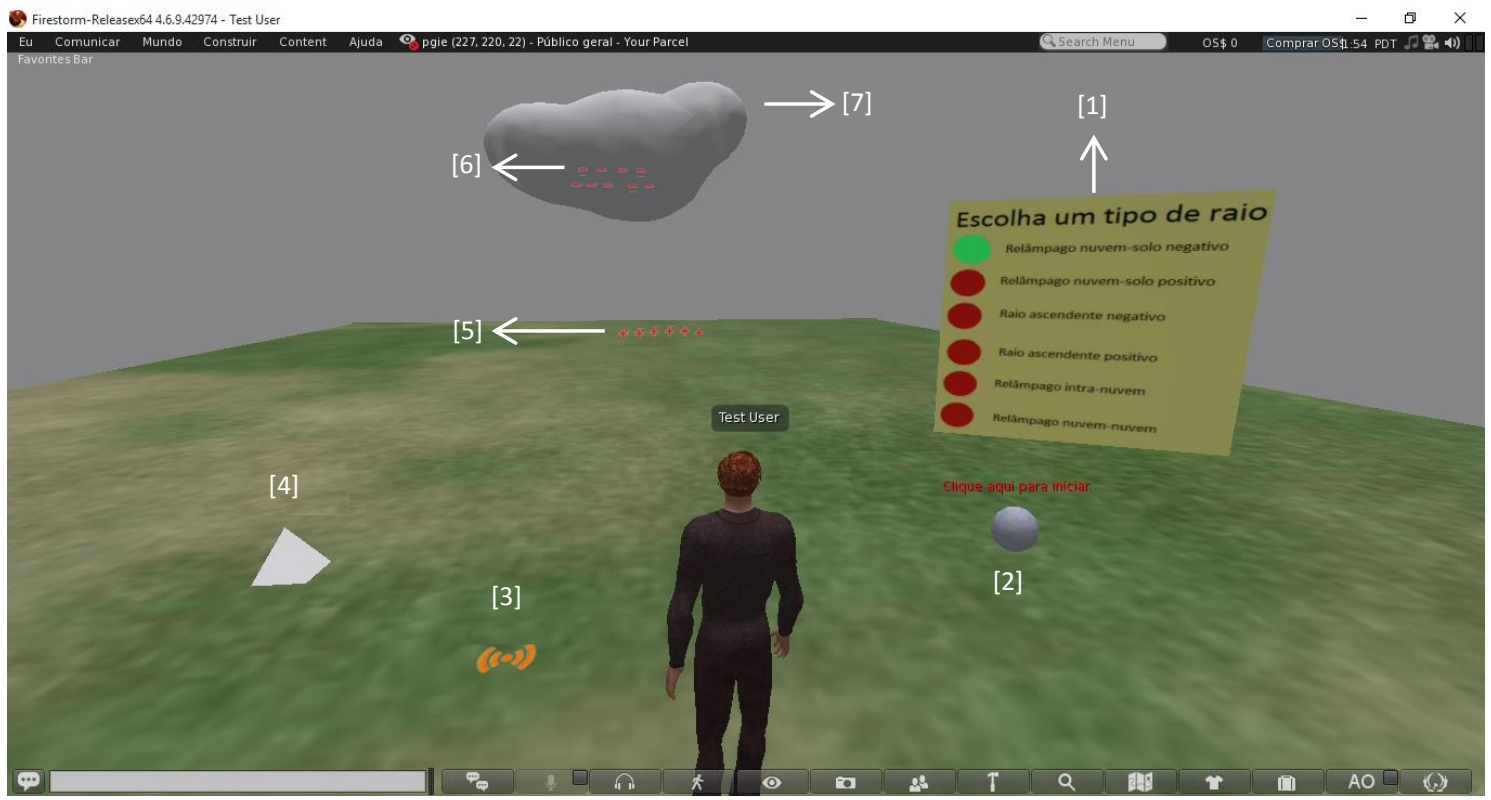

Figura 3: Experimento com sensores geográfico e de interação.

A experiência de relâmpagos tem o seguinte funcionamento básico: quando o senso de geográfico detecta a presença do avatar, ele registra sua chegada e saída desta experiência no BD. O código que executa este registro é apresentado na Figura 4.

chamadaHTTP $=$

11HTrPRequest ("http://127,0 0,1/opensim/grava trilha,php?workflow="+workflow+" \&local="+local+" \&datahora="+ dataHora+" \&idavatar="+id_avatar+" \&nomeavatar="+nome_avatar+" £presenca="+presenca, [HTTP__EETHOD, "GET"], ,") ;

Figura 4: Gravação das informações coletadas pelos sensores geográficos em um banco de dados.

Após sua chegada, o aluno escolhe uma das 6 opções disponíveis no menu (detalhe 1 da Figura 3), neste caso "Relâmpago nuvem-solo negativo", definida como opção 1. O objeto menu envia duas mensagens: (I) para as cargas elétricas, que devem ajustar seu sinal para positivo ou negativo, de acordo com a opção escolhida (detalhes 5 e 6 da Figura 3) e (II) para o sensor de interação (detalhe 4 da Figura 3), informando a identificação do avatar, a opção selecionada pelo aluno, o nome do objeto que foi acionado e o nome da experiência que está sendo realizada.

A seguir o aluno deve clicar no objeto esfera (detalhe 2 da Figura 3) para iniciar a experiência. O objeto esfera envia duas mensagens: (A) para as cargas elétricas, as quais devem iniciar a animação, sendo que neste caso as cargas negativas irão surgindo e se posicionando na direção das cargas positivas e (B) para o sensor de interação (detalhe 4 da Figura 3), que recebe a informação de que o aluno acionou o experimento.

Ao encerrar a experiência o aluno pode escolher repetir a mesma opção, executar uma nova ou ainda sair do local. Todas as situações serão registradas conforme descrito acima. A Figura 5 apresenta o código que está embutido no objeto sensor de interação. 
11HTTPRequest ("http://127,0 0.1/opensim/grava_interacao. php?avatar="+id_avatart"£opcao="+opcaoselt" £objetoemissor"t

objemissort" «experimento="texperimento, [HTMP_METHOD, "GET"]," ") ;

Figura 5: Experimento com sensores geográfico e de interação.

A gravação dos dados dos sensores pressupõe a criação de um banco de dados com tabelas apropriadas para o armazenamento das informações. Nesta proposta os dados são gravados através de comandos HTTP que executam páginas PHP, permitindo a independência de estrutura hardware e software.

\section{Conclusões}

Este trabalho descreveu os conceitos de aprendizagem experiencial e aprendizagem significativa, os tipos e classes de laboratórios virtuais; mostrou os mundos virtuais 3D e seus recursos para a interação entre o avatar do aluno e seus objetos, descreveu as áreas de mineração de dados educacionais e Learning Analytics.

Foi apresentada a possibilidade de construção de laboratórios nos mundos virtuais 3D que consideram a taxonomia de engajamento estendida, de forma que os objetos em si e o laboratório como um todo, permitam a aprendizagem experiencial e significativa. Descreveu uma área de pré-experimento, onde o aluno pode interagir com o material educacional controlando sua visualização e inserindo dados, obtendo ou revisando conhecimento; e uma área de pós-experimento, onde aluno pode verificar o conhecimento obtido na área de pré-visualização e no experimento, por meio de respostas à objetos de avaliação.

Foi proposta uma forma para identificar o nível de engajamento do aluno nas experiências através da criação de sensores geográficos e de interação, os quais permitem registrar as ações do estudante nos locais do laboratório, bem como a sua interação com os objetos e equipamento da experiência.

Assim, conclui-se que a construção de laboratórios nos mundos virtuais 3D que permitam a aprendizagens experiencial e significativa é possível, pois a experiência apresentada permite a alteração da visualização durante sua apresentação, bem como a sua manipulação direta, indicando o nível 6 na taxonomia de Myller et al. (2009).

Como trabalhos futuros, serão aplicadas técnicas de mineração de dados educacionais e consequentemente Learning Analytics, para a identificação de alunos em "risco" e desencadeamento de operações de "resgate" (atividades definidas para próxima etapa do projeto de tese). Ainda, é necessário estudar a construção ferramentas que permitam atingir os níveis superiores da taxonomia de Myller et al. (2009).

\section{Referências Bibliográficas}

AMARAL, Érico; ÁVILA, Bárbara ; ZEDNIK, Herik; TAROUCO, Liane. Laboratório Virtual de Aprendizagem: Uma Proposta Taxonômica. Revista Novas Tecnologias na Educação - RENOTE. v. 9, n. 2. 2011.

AMARAL, Érico; ÁVILA, Barbara G.; TAROUCO, Liane M. R. Aspectos teóricos e práticos da implantação de um laboratório virtual no OpenSim. $\mathbf{2 3}^{\mathbf{0}}$ Simpósio Brasileiro de Informática na Educação (SBIE 2012). 2012. Disponível em: http://www.br-ie.org/pub/index.php/sbie/article/view/1763.

AUER, M.; PESTER, A.; URSUTIU, D.; SAMOILA, D. Distributed Virtual and Remote Labs in Engineering, IEEE International Conference On Industrial Technology (ICIT), Maribor, Slovenia, 2003, v. 2, p. 1208 - 1213.

ÁVILA, Bárbara ; AMARAL, Érico M. H.; TAROUCO, Liane M. R. Implementação de Laboratórios Virtuais no metaverso OpenSim. Revista Novas Tecnologias na Educação - RENOTE. v. 11, n. 1. 2013. 
ÁVILA, Bárbara; AMARAL, Érico M.H.; MÜLLER, Thaísa J.; TAROUCO, Liane. Validação de Laboratórios Virtuais de Aprendizagem baseada em uma visão Taxonômica. Revista Novas Tecnologias na Educação - RENOTE. v. 10, n. 2. 2012. CAMILLERI, V.; FREITAS, S. de; MONTEBELLO, M.; MCDONAGH-SMITH, P. A case study inside virtual worlds: use of analytics for immersive spaces. In Proceedings of the Third International Conference on Learning Analytics and Knowledge, ACM, New York, Pag. 230-234, 2013. Disponível em:http://dl.acm.org/citation.cfm?id=2460296.2460341\&coll=DL\&dl=ACM\&CFID=5 58250904\&CFTOKEN=38872506, Acesso em: 24 Abr. 2015.

COSTA, E.; BAKER, R.S.J.d.; AMORIM, L.; MAGALHÃES, J.; MARINHO, T. Mineração de Dados Educacionais: Conceitos, Técnicas, Ferramentas e Aplicações, Jornada de Atualização em Informática na Educação - JAIE 2012. Disponível em: http://www.br-ie.org/pub/index.php/pie/article/view/2341, Acesso em: 27 Out. 2015. CRUZ-BENITO, J.; THERON, R.; GARCIA-PEÑALVO, F.J.; MADERUELO, C.; PEREZ-BLANCO, J.S.; ZAZO, H.; MARTIN-SUAREZ, A. Monitoring and feedback of learning processes in virtual worlds through analytics architectures. In: Iberian Conference on Information Systems and Technologies. 2014. Disponível em: http://ieeexplore.ieee.org/xpls/abs_all.jsp?arnumber=6877097, Acesso: 05 Mai. 2015. FEISEL, L.D.; ROSA A.J.The Role of the Laboratory in Undergraduate Engineering Education, Journal of Engineering Education. 2005. Vol. 94. Pg. 121-130.

FERGUSON, R. The State Of Learning Analytics in 2012: A Review and Future Challenges, Technical Report KMI-12-01, Knowledge Media Institute, The Open University, 2012, Disponível em: http://kmi.open.ac.uk/publications/techreport/kmi-1201. Acessado em: 20 Mai. 2015.

FERGUSON, R.; SHUM, S. B. Social learning analytics: five approaches. Proceedings of the 2nd International Conference on Learning Analytics and Knowledge. pag.23-33, ACM, New York, 2012. Disponível em: http://dl.acm.org/citation.cfm?id=2330616, Acesso em: 03 Mai. 2015.

FERNÁNDEZ-GALLEGO, B.; LAMA, M.; VIDAL, J.C.; MUCIENTES, M. Learning Analytics Framework for Educational Virtual Worlds, Procedia Computer Science, Volume 25, 2013, pag. 443-447, Disponível em: www.sciencedirect.com/ science/article/pii/S1877050913012611, Acesso em: 13 Mar. 2015.

GREIS, L. K.; REATEGUI, E.; MARQUES, T. B. I. Un Simulador de Fenómenos Físicos para los Mundos Virtuales / A physical phenomena simulator for virtual worlds. Revista Latinoamericana de Tecnología Educativa. v. 12, núm. 1. 2013. Pg. 51-62, Disponível: 〈http://campusvirtual.unex.es/revistas/index.php/relatec/article/view/893〉.

HOWLAND, J.; JONASSEN, D.; MARRA, R. Meaningful Learning with Technology, 3rd edition, Pearson Education: Upper Saddle River, New Jersey, 2008. IEDMS, International Educational Data Mining Society. Disponível em: http://www.educationaldatamining.org/, Acesso em: 27 Jun. 2015.

JONASSEN, David H.; PECK, Kyle L.; WILSON, Brent G. Learning with Technology - A Construtivist Perspective. Prentice Hall, 1999.

KICKMEIER-RUST, M.D.; ALBERT, D. Learning Analytics to Support the Use of Virtual Worlds in the Classroom. Human-Computer Interaction and Knowledge Discovery in Complex, Unstructured, Big Data. Vol. 7947, pag. 358-365. 2013. Disponível em: http://link.springer.com/chapter/10.1007\%2F978-3-642-39146-0_34\# Acessado em: 16 Abr. 2015.

KLASTRUP, Lisbeth. A Poetics of Virtual Worlds. Melbourne DAC 2003. Melbourne. 2003, Disponível em <http://hypertext.rmit.edu.au/dac/papers/>. Acesso: 22 mai. 2013. 
KOLB, David A.; BOYATZIS, Richard E.; MAINEMELIS, Charalampos. Experiential Learning Theory: Previous Research and New Directions. 31 ago. 1999. Disponível em: <http://www.d.umn.edu/ kgilbert/educ5165731/Readings/experiential-learning-theory.pdf>. Acesso em: 11 Mai. 2013.

KOLB, David. A. Experiential Learning: Experience as the Source of Learning and Development. Englewood Cliffs, N.J.: Prentice-Hall, 1984.

MATTAR, João. O Uso do Second Life como ambiente de Aprendizagem. Revista Fonte, 2008.

MEC. Ciências da natureza, matemática e suas tecnologias. Brasília : Ministério da Educação, Secretaria de Educação Básica, 2006. 135 p.

MEC. PCN-EM Orientações Educacionais Complementares aos Parâmetros Curriculares Nacionais: Ciências da Natureza, Matemática e suas Tecnologias. Disponível em: <http://portal.mec.gov.br/seb/arquivos/pdf/CienciasNatureza.pdf>, Acessado em: 24 Out. 2015.

MOREIRA, Marco Antônio. Teorias de aprendizagem. São Paulo: EUP, 1999. MYLLER, N.; BEDNARIK, R.; SUTINEN, E.; BEN-ARI, M. Extending the Engagement Taxonomy: Software Visualization and Collaborative Learning. ACM Transactions on Computing Education. Vol. 9, N. 1, 2009. Disponível em: http://dl.acm.org/citation.cfm?id=1513600. Acesso: 23 Abr. 2014.

NELSON, B.C.; ERLANDSON, B.E. Design for Learning in Virtual Worlds: Interdisciplinary Approaches Educational Technology. New York: Routledge. 2012. OPENSIM. OpenSim. Disponível: http://opensimulator.org/. Acesso em: 07 ago. 2013. SANTAELLA, Lucia. Cultura e artes do pós-humano. São Paulo: Paulus. 2003.

SCHAF, Frederico Menine. Arquitetura modular para ambientes virtuais de ensino de automação com suporte à realidade mista e colaboração. Universidade Federal do Rio Grande do Sul. Escola de Engenharia. Tese. 2011. Disponível em: <http://www.lume.ufrgs.br/handle/10183/28954. 2011>. Acesso em: 15 mar. 2013.

SECOND LIFE. Second Life Wiki. Disponível em: http://wiki.secondlife. com/wiki/Help:Getting_started_with_LSL. Acesso em 13 Ago. 2013.

SIEMENS, G. What are learning analytics?, Disponível em: http://www.elearnspace. org/blog/2010/08/25/what---are---learning---analytics/. Acessado em: 20 Mai. 2015.

TIBOLA, Leandro R.; TAROUCO, Liane M. R.; PASSERINO, Liliana M. A possibilidade de identificação da ação mediada nos mundos digitais virtuais 3D. Revista Novas Tecnologias na Educação - RENOTE. v. 11, n. 3. 2013.

VOSS, G.; MEDINA, R.; AMARAL, É.; ARAÚJO, F.; NUNES, F.; OLIVEIRA, T. Proposta de Utilização de Laboratórios Virtuais para o Ensino de Redes de Computadores: Articulando Ferramentas, Conteúdos e Possibilidades. Revista Novas Tecnologias na Educação - RENOTE. v. 10, n. 2. 2012.

WOLFF, A.; ZDRAHAL, A.; NIKOLOV, A.; PANTUCEK, M. Improving retention: predicting at-risk students by analysing clicking behaviour in a virtual learning environment. In: Proceedings of the Third International Conference on Learning Analytics and Knowledge, ACM, New York, pag. 145-149. 2013. Disponível em: http://dl.acm.org/citation.cfm?id=2460324, Acesso em: 03 Mai.2015.

WORSLEY, Marcelo; BLIKSTEIN, Paulo. Towards the development of multimodal action based assessment. In Proceedings of the Third International Conference on Learning Analytics and Knowledge, ACM, New York, pag. 94-101, 2013, Disponível em: http://dl.acm.org/citation.cfm?id=2460315, Acesso em 03 Mar. 2015.

ZEDNIK, Herik; AMARAL, Érico; ÁVILA, Bárbara; TAROUCO, Liane. VEGA Implementando um Laboratório Virtual Imersivo no OpenSim. Revista Novas Tecnologias na Educação - RENOTE. v. 10, n. 1. 2012. 